EPJ manuscript No.

(will be inserted by the editor)

\title{
Medium Modification of the Proton Form Factor
}

\author{
Steffen Strauch for the Jefferson Lab E93-049 collaboration
}

Department of Physics, The George Washington University, Washington, DC 20052, USA

Received: date / Revised version: date

\begin{abstract}
I argue that the double ratio of proton-recoil polarization-transfer coefficients, $P_{x}^{\prime}$ and $P_{z}^{\prime}$, of the quasielastic ${ }^{4} \mathrm{He}\left(\boldsymbol{e}, e^{\prime} \boldsymbol{p}\right)^{3} \mathrm{H}$ reaction with respect to the elastic ${ }^{1} \mathrm{H}\left(\boldsymbol{e}, e^{\prime} \boldsymbol{p}\right)$ reaction is sensitive to possible medium modifications of the proton form factor in ${ }^{4} \mathrm{He}$. Recent measurements at both Mainz and Jefferson Lab of this double ratio at four-momentum transfers squared between between $0.4(\mathrm{GeV} / \mathrm{c})^{2}$ and 2.6 $(\mathrm{GeV} / \mathrm{c})^{2}$ are discussed. I show that the data challenge state-of-the-art conventional meson-nucleon calculations, as these are unable to describe the results. The data hint at the need to include medium modifications of the proton form factor, as predicted by a quark-meson-coupling model, in the calculations. A recently approved follow-up experiment at a $Q^{2}$ of $0.8(\mathrm{GeV} / \mathrm{c})^{2}$ and $1.3(\mathrm{GeV} / \mathrm{c})^{2}$ with unprecedented precision will provide one of the most stringent tests of the applicability of various calculations.
\end{abstract}

PACS. 25.30.Dh Inelastic electron scattering to specific states $-24.70 .+\mathrm{s}$ Polarization phenomena in reactions - 14.20.Dh Protons and neutrons - 13.40.Gp Electromagnetic form factors - 13.88.+e Polarization in interactions and scattering $-27.10 .+\mathrm{h} \quad A \leq 5$

\section{Introduction}

(1) The standard nuclear-physics model describes nuclei as 1 clusters of protons and neutrons held together by a strong force mediated by meson exchange. The success of the nuclear shell model demonstrates that this approxima- tion has been highly effective. A recent example is the very good description of the differential cross section and left-right asymmetry in the ${ }^{16} \mathrm{O}\left(e, e^{\prime} p\right)$ reaction [1] with a fully relativistic distorted-wave impulse approximation (RDWIA).

However, protons and neutrons are not the fundamental entities of the underlying theory, quantum chromodynamics. At nuclear densities of about 0.17 nucleons $/ \mathrm{fm}^{3}$, nucleon wave functions have significant overlap. In the chiral limit, one expects nucleons to lose their identity altogether and nuclei to make a transition to a quark-gluon plasma. For that reason, one may expect that, for some observables, the free-nucleon approximation is a highly uneconomical approach. The use of medium-modified nucleons as quasiparticles might be a better choice. To demonstrate that the interpretation of the experimental data is more efficient in terms of medium modifications of nucleon form factors, it is required to have excellent control of the inherent many-body effects, such as meson-exchange currents (MEC) and isobar configurations (IC). In addition, when probing nuclear structure, one has to deal with finalstate interactions (FSI).

In unpolarized $\mathrm{A}\left(e, e^{\prime} p\right)$ experiments involving lightand medium-mass nuclei, deviations were observed in the longitudinal/transverse character of the nuclear response compared with the free-proton case $2,3,4$. Below the two- nucleon-emission threshold, these deviations were originally interpreted as changes in the nucleon form factors within the nuclear medium. However, strong-interaction effects on the ejected proton (final-state interactions) later also succeeded in explaining the observed effect [5]. Still, tantalizing hints of medium effects remain for unpolarized longitudinal/transverse separations in the ${ }^{4} \mathrm{He}\left(\mathrm{e}, \mathrm{e}^{\prime} \mathrm{p}\right)^{3} \mathrm{H}$ reaction 6.7.7.

A reaction which is believed to have minimal sensitivity to reaction-mechanism effects is polarization transfer in quasielastic nucleon knockout (see, e.g., [8]). The polarization-transfer observables are sensitive to the properties of the nucleon in the nuclear medium, including possible modification of the nucleon form factor and/or spinor. This can be seen from free electron-nucleon scattering, where the ratio of the electric to magnetic Sachs form factors, $G_{E}$ and $G_{M}$, is given by $[9]$ :

$$
\frac{G_{E}}{G_{M}}=-\frac{P_{x}^{\prime}}{P_{z}^{\prime}} \cdot \frac{E_{e}+E_{e^{\prime}}}{2 m_{p}} \tan \left(\theta_{e} / 2\right) .
$$

Here, $P_{x}^{\prime}$ and $P_{z}^{\prime}$ are the transferred polarizations, transverse and longitudinal to the proton momentum (see [10]). The beam energy is $E_{e}$, the energy (angle) of the scattered electron is $E_{e^{\prime}}\left(\theta_{e}\right)$, and $m_{p}$ is the proton mass. This relation was used to extract $G_{E} / G_{M}$ for the proton (most recently in 11 12 13).

In the following, the results of recent ${ }^{4} \mathrm{He}\left(\boldsymbol{e}, e^{\prime} \boldsymbol{p}\right)^{3} \mathrm{H}$ experiments to study the proton knock-out process will be discussed. In these experiments ${ }^{4} \mathrm{He}$ was chosen as target because of its high density and relatively simple structure, which allows for RDWIA and microscopic calculations. 
The kinematics of these experiments, quasielastic scattering at low missing momentum with symmetry about the three-momentum-transfer direction, minimize conventional many-body effects, as will be demonstrated below.

\section{Experiments}

The first ${ }^{4} \mathrm{He}$ polarization-transfer measurement was performed at the Mainz microtron (MAMI) at a fourmomentum squared $Q^{2}=0.4(\mathrm{GeV} / \mathrm{c})^{2}$ 14. More recently these measurements were extended to $Q^{2}=0.5$, 1.0, 1.6, and $2.6(\mathrm{GeV} / \mathrm{c})^{2}$ in experiment E93-049 at Jefferson Lab Hall A 15 16. Since these experiments were designed to detect differences between the in-medium polarizations compared to the free values, both ${ }^{4} \mathrm{He}$ and ${ }^{1} \mathrm{H}$ targets were employed (due to beam-time constraints, only ${ }^{4} \mathrm{He}$ data were acquired at $\left.Q^{2}=2.6(\mathrm{GeV} / \mathrm{c})^{2}\right)$. The induced polarization, $P_{y}$, was also measured in the JLab experiment 16 .

In each experiment, two high-resolution spectrometers were used, one to detect the scattered electron and one to detect the recoiling proton. The latter was equipped with a focal-plane polarimeter (FPP). The proton-recoilpolarization observables were extracted by means of the maximum-likelihood technique, utilizing the azimuthal distribution of protons scattered off the graphite analyzer in the FPP.

The results of the measurements are expressed in terms of the polarization-transfer double ratio, in which the helium polarization ratio is normalized to the hydrogen polarization ratio measured in the identical setting:

$$
R=\frac{\left(P_{x}^{\prime} / P_{z}^{\prime}\right)^{4} \mathrm{He}}{\left(P_{x}^{\prime} / P_{z}^{\prime}\right)^{1} \mathrm{H}}
$$

As a cross-check, the hydrogen results were also used to extract the free-proton form-factor ratio $G_{E} / G_{M}$, which was found to be in excellent agreement with previous data 11,12.

Nearly all systematic uncertainties cancel in $R$ : the polarization-transfer observables are to first order independent of instrumental asymmetries in the FPP, and their ratio is independent of the electron-beam polarization and carbon analyzing power. Small systematic uncertainties are predominantly due to uncertainties in the spin transport through the proton spectrometer.

\section{Results}

Figure 1 shows the results for $R$ at $Q^{2}=1.0(\mathrm{GeV} / \mathrm{c})^{2}$ as a function of missing momentum. The five data points correspond to five bins in the acceptance within the single experimental setting. Negative values of missing momentum correspond to the recoiling nuclei having a momentum component antiparallel to the direction of the threemomentum transfer.

The data are compared with several calculations, which were averaged over the experimental acceptance.

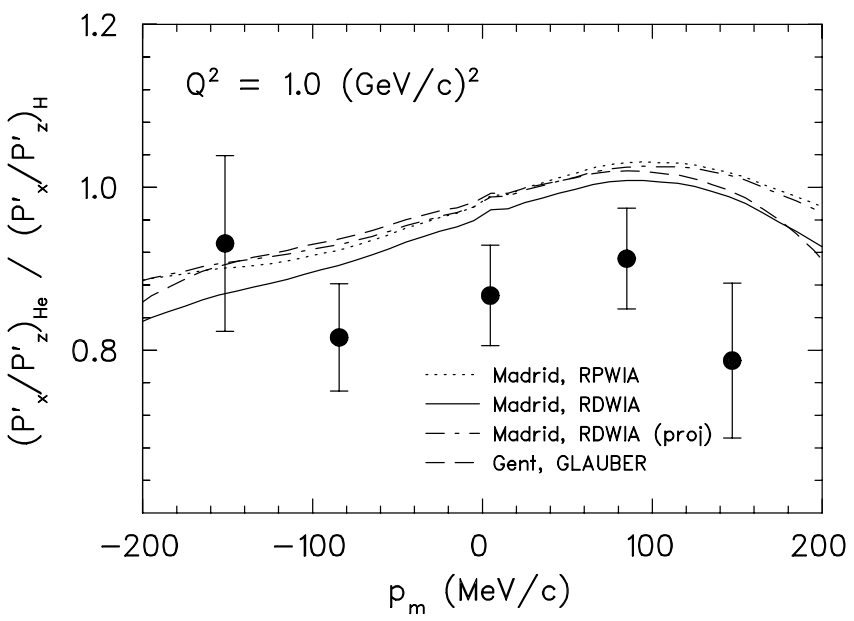

Fig. 1. Polarization-transfer double ratio in ${ }^{4} \mathrm{He}\left(\boldsymbol{e}, e^{\prime} \boldsymbol{p}\right)^{3} \mathrm{H}$ at $Q^{2}=1.0(\mathrm{GeV} / \mathrm{c})^{2}$ as a function of missing momentum. The data [16] are compared to calculations by the Madrid [17] and Gent 18, 19 groups. Error bars are statistical only; systematic uncertainties are much smaller.

In the relativistic calculations by the Madrid group [17, the Coulomb gauge and the current operator $c c 1$ [20] were used. The dotted line is a result of a calculation in planewave impulse approximation (RPWIA). This calculation predicts $R \approx 1$ at $p_{m}=0$. It overpredicts the data by about $10 \%$, although the missing-mass distribution of the data is reasonably well described. The relativistic distorted-wave impulse approximation (RDWIA) gives a smaller value of $R(\approx 3 \%)$ than the RPWIA but still overpredicts the data (solid curve). The calculation uses the MRW optical potential of 21. The main difference in the results between this full calculation and the RPWIA is due to the enhancement of the negative energy components of the relativistic bound and scattered proton wave functions. This can be demonstrated by projecting the wave functions over positive-energy states (dash-dotted curve) resulting in essentially the same polarization-transfer ratio as given by RPWIA. Also, a Glauber calculation by the Gent group [18,19] (long-dashed line) is unable to account for the data.

The induced polarization $P_{y}$ in the $\left(e, e^{\prime} \boldsymbol{p}\right)$ reaction is identically zero, in the absence of FSI effects (in the onephoton-exchange approximation). This observable thus constitutes a stringent test of various FSI calculations. Figure 2 shows the results, corrected for (small) false asymmetries, as a function of $Q^{2}$. One sees that the induced polarizations are small for all measured $Q^{2}$ values.

The dashed and dot-dashed curves constitute RDWIA calculations by the Madrid group [17 with the MRW 21] and RLF 22] relativistic optical potentials. For the induced-polarization case, the RDWIA curves do not depend on the nucleon form-factor model chosen in the calculations. Figure 2 confirms the expected smallness of the induced polarizations, and seems to indicate a reasonable agreement with the RDWIA calculation.

However, due to the relatively large systematic uncertainties caused by possible false asymmetries in the FPP, 


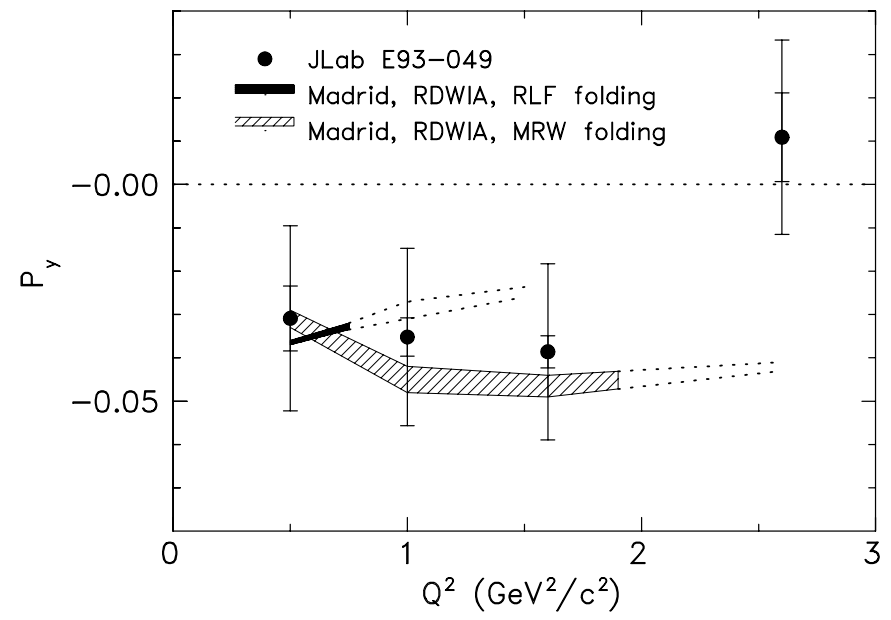

Fig. 2. Measured values of the induced polarizations for the ${ }^{4} \mathrm{He}\left(e, e^{\prime} \boldsymbol{p}\right)^{3} \mathrm{H}$ reaction [16. The inner uncertainty is statistical only, the total uncertainty includes a systematic uncertainty of \pm 0.02 , due to the imperfect knowledge of the false asymmetries. The hatched areas show the results for different RDWIA calculations [17] with the MRW [21] and RLF [22] relativistic optical potentials. All theoretical curves are averaged over the experimental acceptance. Dotted curves indicate calculations in which the optical potentials were extrapolated beyond the region of validity.

the test is not conclusive. A far more detailed study of the induced polarization can be made with data expected from JLab experiment E03-104 23].

\section{Discussion}

The disagreement between the data and the RDWIA calculations for the polarization double ratio $R$ is puzzling, since, as mentioned earlier, these relativistic calculations provide good descriptions of, e.g., the induced polarizations measured at Bates in the ${ }^{12} \mathrm{C}\left(e, e^{\prime} \boldsymbol{p}\right)$ reaction 24 and of $A_{T L}$ in ${ }^{16} \mathrm{O}\left(e, e^{\prime} p\right)$ as previously measured at JLab [1]. To find a possible explanation for that disagreement, a detailed study of model dependencies was performed.

First, the model input in the calculations of the Madrid group was studied. Figure 3(a) shows again the $Q^{2}=$ $1.0(\mathrm{GeV} / \mathrm{c})^{2}$ polarization-transfer double-ratio data. The hatched area indicates the range of different RDWIA calculations. For these calculations, various bound-state wave functions, current operators ( $c c 1$ and $c c 2$ ), and optical potentials were chosen. The optical potentials include the MRW folding parameters [21] and the RLF folding parameters 22. The latter are valid only up to $T_{\text {lab }} \approx 400 \mathrm{MeV}$, and thus are already beyond its regime of validity for $Q^{2}$ of $1.0(\mathrm{GeV} / \mathrm{c})^{2}$. Different optical potentials were constructed for each parameter set based upon the experimental ${ }^{3} \mathrm{H}$ density, based upon a simple Woods-Saxon density for ${ }^{3} \mathrm{H}$ with the same root-mean-square radius as the experimental one, and, finally, one with an $30 \%$ increased root-mean-square radius, resulting in an unrealistic potential. Different parametrizations of the free-nucleon form

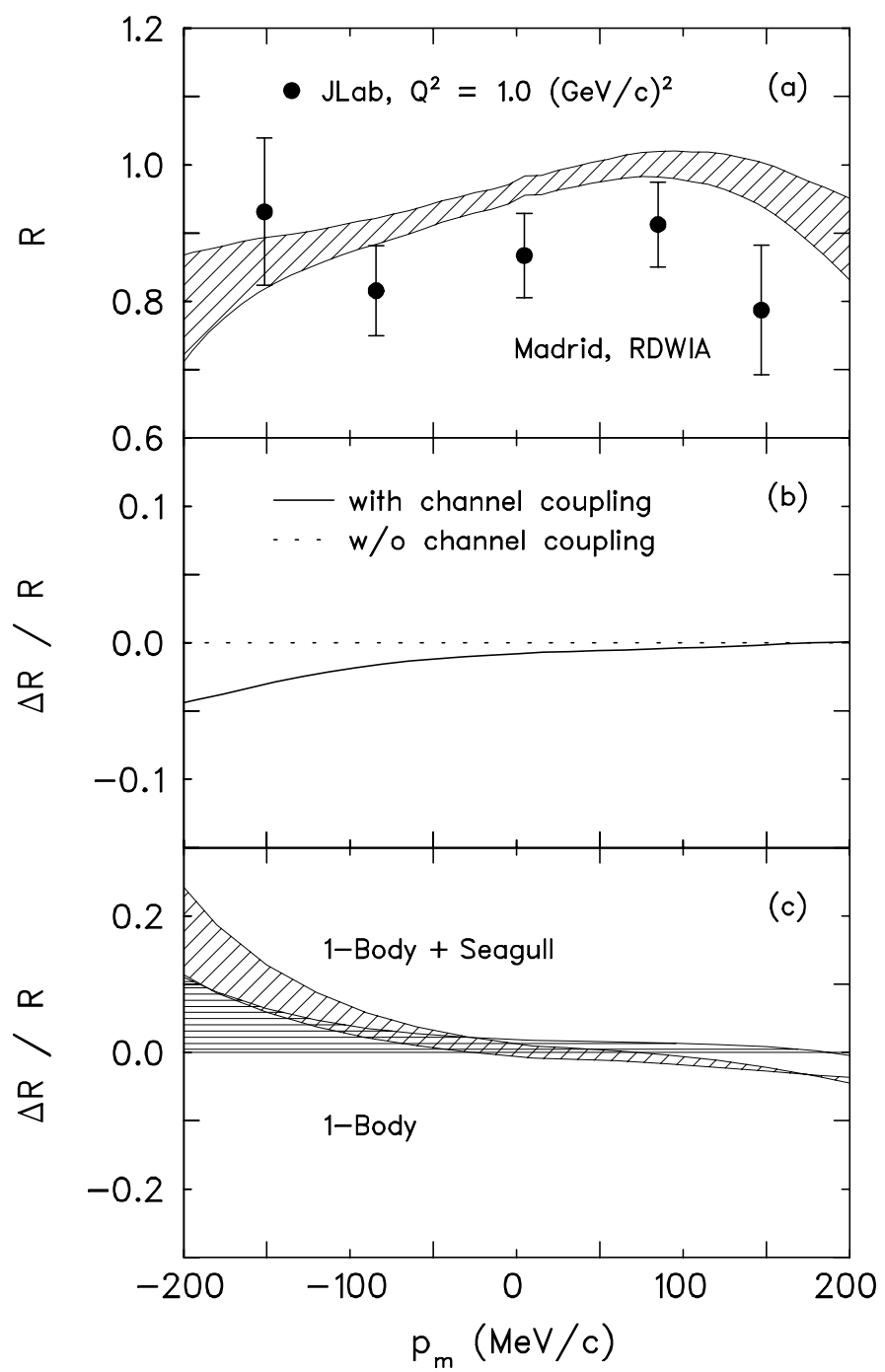

Fig. 3. Study of model sensitivities to the polarizationtransfer double ratio in the ${ }^{4} \mathrm{He}\left(\boldsymbol{e}, e^{\prime} \boldsymbol{p}\right)^{3} \mathrm{H}$ reaction at $Q^{2}=1.0$ $(\mathrm{GeV} / \mathrm{c})^{2}$. (a) E93-049 polarization transfer double ratio at $Q^{2}=1.0(\mathrm{GeV} / \mathrm{c})^{2}$ along with a PWIA and different RDWIA calculations of the Madrid group [17. (b) Relative difference between the polarization transfer ratio including channel coupling and without channel coupling. The calculation follows the approach of [25]. (c) Differences in the polarization double ratios relative to $R_{\mathrm{cc} 1}$ in the ${ }^{4} \mathrm{He}\left(\boldsymbol{e}, e^{\prime} \boldsymbol{p}\right)^{3} \mathrm{H}$ reaction as a function of missing momentum (perpendicular kinematics). Plotted are results for different de Forest current operators with and without the MEC seagull diagram. The 1-body $c c 1$ result serves as a baseline. Calculation courtesy of Meucci [26].

factors were also used. To a very good approximation, the ratio $R$ is independent of such variations, if the same parametrization is used in the ${ }^{4} \mathrm{He}$ and ${ }^{1} \mathrm{H}$ calculations. It is evident from Fig. 3(a) that there is hardly any sensitivity in the polarization-transfer ratio at low missing momentum (within the models examined) to the different and partly extreme choices of optical potential, one-body current operator, and bound-state wave function.

Next, the effect of channel coupling on $R$ for the ${ }^{4} \mathrm{He}\left(\boldsymbol{e}, e^{\prime} \boldsymbol{p}\right)^{3} \mathrm{H}$ reaction was estimated. Recently Kelly [25] 
investigated the sensitivity of recoil-polarization observables in $A\left(\boldsymbol{e}, e^{\prime} \boldsymbol{p}\right) B$ reactions to channel coupling in finalstate interactions (for ${ }^{12} \mathrm{C}$ and ${ }^{16} \mathrm{O}$ ). In these studies it was found that polarization-transfer observables for proton knockout with modest missing momentum appear to be quite insensitive to details of the final-state interaction, including channel coupling. Following the approach of Kelly 25, the effect of channel coupling was estimated for the ${ }^{4} \mathrm{He}\left(\boldsymbol{e}, e^{\prime} \boldsymbol{p}\right)^{3} \mathrm{H}$ reaction by calculating the relative difference between the polarization transfer ratio with and without channel coupling. Couplings between the proton $1 s_{1 / 2}$ and neutron $1 s_{1 / 2}$ states were considered. Figure 3(b) shows the results for a four-momentum transfer of $Q^{2}=1.0(\mathrm{GeV} / \mathrm{c})^{2}$. The effect is minimal, and on average is of the order of $1 \%-2 \%$.

Finally, the effects of two-body interactions were considered. Available model calculations indicate that these contributions are smallest in quasielastic, parallel kinematics and at low missing momentum; the kinematics of the experiments discussed here. In a recent work, the Pavia group has studied meson-exchange currents in a relativistic model for electromagnetic one-nucleon emission [26] (for ${ }^{12} \mathrm{C}$ and ${ }^{16} \mathrm{O}$ ). Meucci has provided us with similar calculations for ${ }^{4} \mathrm{He}$; these results are shown in Fig. B( $(\mathrm{c})$ for $Q^{2}=1.0(\mathrm{GeV} / \mathrm{c})^{2}$ as relative differences with respect to the one-body $c c 1$ calculation. The horizontally hatched band covers the range of calculations with three different one-body currents $(c c 1, c c 2, c c 3)$. The calculations confirm that, at low missing momentum, ambiguities due to the choice of the 1-body current $(c c 1, c c 2, c c 3)$ are small, of the order of $3 \%$. The inclusion of the two-body current in the form of the seagull diagram with one-pion exchange (diagonally hatched band) has an asymmetric effect on the polarization ratio about $\mathbf{p}_{m}=0$; the effect of MEC is also small. It reduces the polarization transfer ratio by about $2 \%$ on average. In addition, these calculations predict the effect of MEC to decrease with increasing four-momentum transfer (not shown).

Also the results of the microscopic calculations of Laget [8] for $Q^{2}=0.4(\mathrm{GeV} / \mathrm{c})^{2}$ and $0.5(\mathrm{GeV} / \mathrm{c})^{2}$ were found to be nearly identical to the RPWIA results (see Fig. 5) indicating that reaction mechanisms like MEC, IC, or charge exchange do not contribute significantly to $R$ in the present kinematics.

Does the present failure of state-of-the-art calculations to describe $R$ indicates a breakdown of standard mesonnucleon calculations? Probably not. It is likely that more complete and more complicated many-body calculations eventually will account for the data. Similarly, the observed cross-section scaling in deuteron photodisintegration at high energies (see e.g. 27]) does not "prove" a breakdown of meson-nucleon calculations, but rather indicates the onset of a regime where quark-gluon-inspired models may give a more effective basis for the interpretation of the data. In the same way, the observed reduction of $R$ in the ${ }^{4} \mathrm{He}\left(\boldsymbol{e}, e^{\prime} \boldsymbol{p}\right)^{3} \mathrm{H}$ reaction motivates the use of a new approach in the interpretation of the data.

Indeed, a calculation by $\mathrm{Lu}$ et al. [28], using a quarkmeson-coupling (QMC) model, suggests a measurable de- viation from the free-space form-factor ratio over the $Q^{2}$ range $0.0<Q^{2}<2.5(\mathrm{GeV} / \mathrm{c})^{2}$. Note that the calculation is consistent with present constraints on possible medium modifications for both the electric form factor (from the Coulomb Sum Rule, with $\mathrm{Q}^{2}<0.5(\mathrm{GeV} / \mathrm{c})^{2}$ 29,30,31), and the magnetic form factor (from a $y$-scaling analysis 32, for $\left.\mathrm{Q}^{2}>1(\mathrm{GeV} / \mathrm{c})^{2}\right)$, and limits on the scaling of nucleon magnetic moments in nuclei 33. Similar measurable effects have been calculated in the light-front-constituent quark model of Frank et al. 34. Recently, Yakshiev et al. 35] investigated possible modifications to the electromagnetic form factors of the nucleons in the ${ }^{4} \mathrm{He}$ nucleus in the framework of a modified Skyrme model, up to $Q^{2}=0.6$ $(\mathrm{GeV} / \mathrm{c})^{2}$. Furthermore, the group of Miller is presently studying medium effects in the framework of a chiral soliton model for the proton 36 .

However, the notion of medium modification of singleparticle properties like, e.g., the electromagnetic form factors of a nucleon, in a nuclear environment is a purely theoretical concept 37. Thus, distinguishing possible changes in the spatial structure of nucleons embedded in a nucleus from more conventional many-body effects is only possible within the context of a model; presently the RDWIA calculation of the Madrid group.

The RDWIA results are brought into excellent agreement with the data by replacing the free-nucleon form factor with a density dependent, medium-modified form factor based on the QMC model 28] (solid curve in Fig. [4).

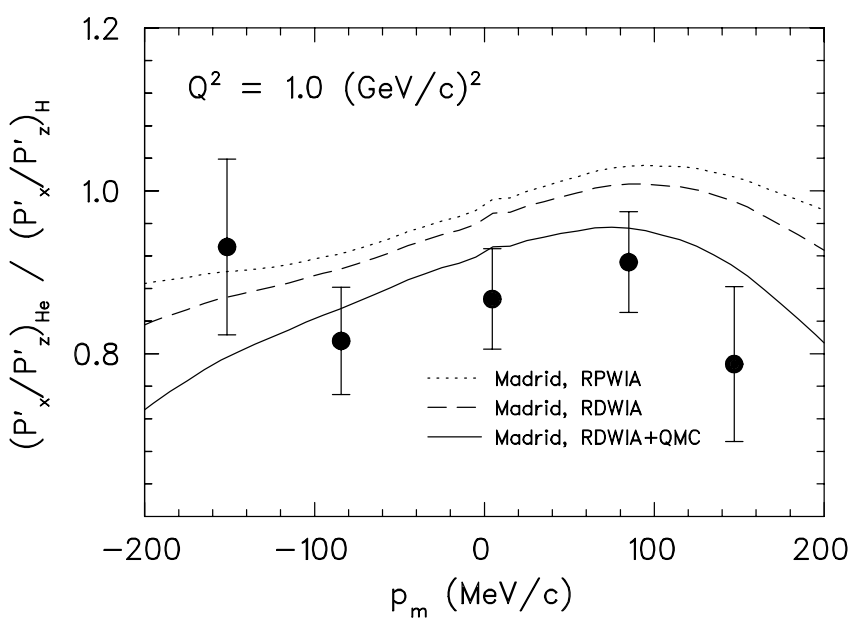

Fig. 4. E93-049 polarization transfer double ratio at $Q^{2}=1.0$ $(\mathrm{GeV} / \mathrm{c})^{2}$ along with a PWIA and different RDWIA calculations of Udias [17.

A summary of all available ${ }^{4} \mathrm{He}\left(\boldsymbol{e}, e^{\prime} \boldsymbol{p}\right)^{3} \mathrm{H}$ data 14,16 is shown in Fig. [5] along with different acceptance-averaged calculations. The polarization-transfer double ratio is plotted as a function of $Q^{2}$. In order not to obscure the result by small kinematical differences between the individual ${ }^{1} \mathrm{H}$ and ${ }^{4} \mathrm{He}$ measurements, data and calculations are shown with $R_{\text {PWIA }}$ as a baseline. The Mainz data point at $Q^{2}=$ $0.4(\mathrm{GeV} / \mathrm{c})^{2}$ closely coincides with the more recent results at $Q^{2}=0.5(\mathrm{GeV} / \mathrm{c})^{2}$ of JLab. 


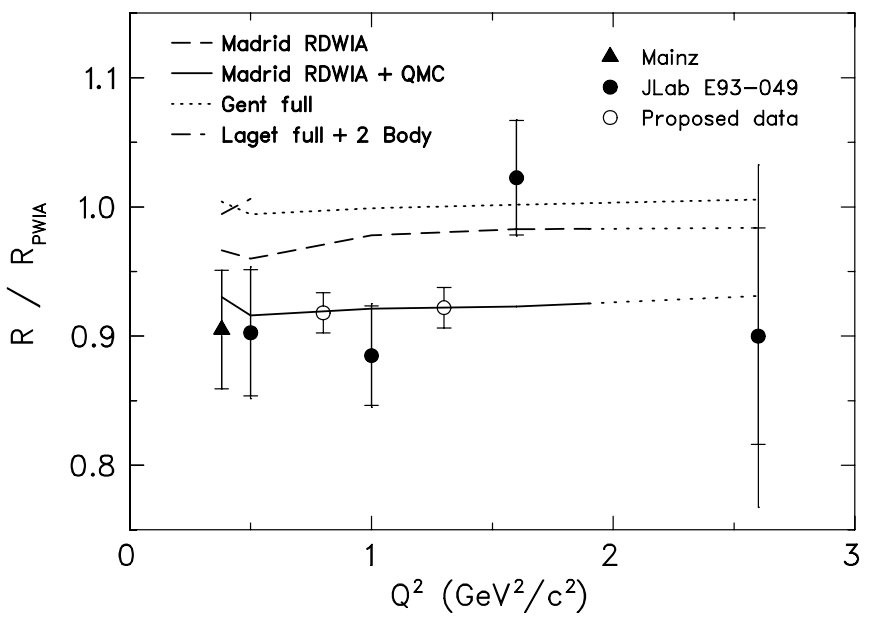

Fig. 5. Superratio $R / R_{\text {PWIA }}$ as a function of $Q^{2}$ (closed circles). The dashed line shows the results of the RDWIA calculation of the Madrid group [17. The dotted line shows the results of the full nonrelativistic model by the Gent group 18 19. The dash-dotted line shows the results of the full calculation, including two-body currents, of Laget [8]. The solid line indicates the full relativistic calculation of the Madrid group 17. including medium modifications as predicted by a quarkmeson-coupling model [28]. For $Q^{2}>1.8(\mathrm{GeV} / \mathrm{c})^{2}$ the Udias calculations maintain a constant relativistic optical potential and are indicated as short-dashed lines. The lines connecting the acceptance-averaged theory calculations are to guide the eye only. The open circles indicate the projected data for experiment E03-104 [23].

On average, the RPWIA calculation (baseline) overestimates the data by $\approx 10 \%$. The dotted line shows the results of the full nonrelativistic model of the Gent group 18, 19. The full calculation, including two-body currents, of Laget 8 is only available for the lower two $Q^{2}$ values (dash-dotted). Both calculations are comparable to the results of RPWIA. The RDWIA calculation (dashed curve) gives a slightly smaller value of $R$ but still overpredicts the data.

The inclusion of medium-modified form factors as predicted by Lu et al. 28] into the RDWIA calculation gives a significantly improved agreement with data, with the exception of the datum at $Q^{2}=1.6(\mathrm{GeV} / \mathrm{c})^{2}\left(\chi^{2}\right.$ per degree of freedom of 1.3 for the five data points), in contrast to the use of free form factors $\left(\chi^{2}\right.$ per degree of freedom of 2.2). All calculations by the Madrid group [17] use the Coulomb gauge, the $c c 1$ current operator as defined in [20], and the MRW optical potential of 21. The $c c 2$ current operator gives higher values of $R$, worsening agreement with the data. As discussed above, various choices for, spinor distortions, current operators, and relativistic corrections affect the theoretical predictions by $\leq 3 \%$, and presently cannot explain the disagreement between the data and the RDWIA calculations.

Also shown in Fig. [5 are the projected data points at $Q^{2}=0.8(\mathrm{GeV} / \mathrm{c})^{2}$ and $1.3(\mathrm{GeV} / \mathrm{c})^{2}$ for the newly approved JLab experiment E03-104 [23]. This experiment will reduce the statistical uncertainties in the double po- larization ratio at each $Q^{2}$ by over a factor of two compared to the previous measurements. These two $Q^{2}$ values were selected since they lie in a region where theoretical calculations are expected to be reliable. This measurement will provide one of the most stringent tests to date of the applicability of conventional meson-nucleon calculations.

Any medium modification of bound-nucleon form factors carries implications for the nuclear EMC effect [36. For example strong constraints are placed on models of the nuclear EMC effect by model-independent relations derived on the basis of quark-hadron duality, which relate the medium modification of the electromagnetic form factors to the modification of the deep-inelastic structure function of a bound proton 38 .

\section{Summary}

Polarization transfer in the quasielastic $\left(e, e^{\prime} p\right)$ reaction is sensitive to possible medium modifications of the boundnucleon form factor, while at the same time largely insensitive to other reaction mechanisms. The ${ }^{4} \mathrm{He}\left(\boldsymbol{e}, e^{\prime} \boldsymbol{p}\right)^{3} \mathrm{H}$ polarization-transfer double-ratio data are not well described by modern relativistic RDWIA calculations using free nucleons as quasiparticles. The data can be efficiently described if medium-modified nucleons are used in the calculations. However, the evidence from the present data is still limited. New high-precision data expected from JLab Hall A should provide a more stringent test of conventional meson-nucleon calculations.

This work was supported in part by the U.S. Department of Energy under grant DE-FG02-95ER40901. Southeastern Universities Research Association (SURA) operates the Thomas Jefferson National Accelerator Facility under U.S. Department of Energy contract DE-AC0584ER40150.

\section{References}

1. J. Gao et al., Phys. Rev. Lett. 84, 3265 (2000).

2. G. van der Steenhoven et al., Phys. Rev. Lett. 57, 182 (1986); 58, 1727 (1987).

3. P. Ulmer et al., Phys. Rev. Lett. 59, 2259 (1987).

4. D. Reffay-Pikeroen et al., Phys. Rev. Lett. 60, 776 (1988).

5. T.D. Cohen, J.W. Van Orden, and A. Picklesimer, Phys. Rev. Lett. 59, 1267 (1987).

6. A. Magnon et al., Phys. Lett. B222, 352 (1989).

7. J.E. Ducret et al., Nucl. Phys. A553, 697c (1993).

8. J.M. Laget, Nucl. Phys. A 579, 333 (1994).

9. A.I. Akhiezer and M.P. Rekalo, Sov. J. Part. Nucl. 3, 277 (1974); R. Arnold, C. Carlson, and F. Gross, Phys. Rev. C 23, 363 (1981).

10. With the initial and final electron momentum $\boldsymbol{k}_{i}$ and $\boldsymbol{k}_{f}$, the coordinate system is given by the unit vectors $\hat{z}=\left(\boldsymbol{k}_{i}-\right.$ $\left.\boldsymbol{k}_{f}\right) /\left|\boldsymbol{k}_{i}-\boldsymbol{k}_{f}\right|, \hat{y}=\left(\boldsymbol{k}_{i} \times \boldsymbol{k}_{f}\right) /\left|\boldsymbol{k}_{i} \times \boldsymbol{k}_{f}\right|$, and $\hat{x}=\hat{y} \times \hat{z}$.

11. M.K. Jones et al., Phys. Rev. Lett. 84, 1389 (2000).

12. O. Gayou et al., Phys. Rev. C 64, 038202 (2001).

13. O. Gayou et al., Phys. Rev. Lett. 88, 092301 (2002).

14. S. Dieterich et al., Phys. Lett. B500, 47 (2001). 
15. Jefferson Lab experiment E93-049 Polarization Transfer in the Reaction ${ }^{4} \mathrm{He}\left(e, e^{\prime} p\right)^{3} \mathrm{H}$ in the Quasi-elastic Scattering Region, R. Ent and P. Ulmer, spokespersons.

16. S. Strauch et al., Phys. Rev. Lett. 91, 052301 (2003).

17. J.M. Udias et al., Phys. Rev. Lett. 83, 5451 (1991); J.A. Caballero, T.W. Donnelly, E. Moya de Guerra, and J.M. Udias, Nucl. Phys. A632, 323 (1998); J.M. Udias and J.R. Vignote, Phys. Rev. C 62, 034302 (2000).

18. J. Ryckebusch, D. Debruyne, W. Van Nespen, and S. Janssen, Phys. Rev. C 60, 034604 (1999).

19. D. Debruyne, Ph.D. thesis, University of Gent (2001).

20. T. de Forest, Nucl. Phys. A392, 232 (1983).

21. J.A. McNeil, L. Ray, and S.J. Wallace, Phys. Rev. C 27, $2123(1983)$.

22. C. J. Horowitz, Phys. Rev. C 31, 1340 (1985).

23. Jefferson Lab experiment E03-104 Probing the Limits of the Standard Model of Nuclear Physics with the ${ }^{4} \mathrm{He}\left(e, e^{\prime} p\right)^{3} H$ Reaction, R. Ent, R. Ransome, S. Strauch, and P. Ulmer, spokespersons.

24. R.J. Woo et al., Phys. Rev. Lett. 80, 456 (1998).

25. J.J. Kelly, Phys. Rev. C 59, 3256 (1999).

26. A. Meucci, C. Giusti, and F.D. Pacati, Phys. Rev. C 66, 034610 (2002), and A. Meucci private communication.

27. R. Gilman and F. Gross, J. Phys. G 28, R37 (2002).

28. D.H. Lu, K. Tsushima, A.W. Thomas, A.G. Williams, and K. Saito, Phys. Lett. B417 (1998) 217 and Phys. Rev. C 60, 068201 (1999).

29. J. Jourdan, Phys. Lett. B353, 189 (1995).

30. J. Morgenstern and Z.-E. Meziani, Phys. Lett. B515, 269 (2001).

31. J. Carlson, J. Jourdan, R. Schiavilla, and I. Sick, Phys. Lett. B553, 191 (2003).

32. I. Sick, Comm. Nucl. Part. Phys. 18, 109 (1988).

33. T.E.O. Ericson and A. Richter, Phys. Lett. B183, 249 (1987).

34. M.R. Frank, B.K. Jennings, and G.A. Miller, Phys. Rev. C 54, 920 (1996).

35. U.T. Yakhshiev, U-G. Meissner, and A. Wirzba, Eur. Phys. J. A 16, 569 (2003).

36. G. Miller, private communication (2003).

37. H. Arenhövel, private communication (2003).

38. W. Melnitchouk, K. Tsushima, and A.W. Thomas, Eur. Phys. J. A 14, 105 (2002). 